\title{
Bases de la modernidad de la arquitectura de Quito del siglo XX
}

\section{Foundations of the modernity of the architecture of Quito of the 20th century}

Recibido: abril 2020

Aceptado: febrero 2021

\section{Resumen}

Los textos de arquitectura que estudian la modernidad en la ciudad de Quito son en su mayoría una recopilación de edificios o de autores y descripciones de sus obras, por lo que no aportan al entendimiento de cómo se representó la modernidad en la ciudad. En consecuencia, se carece de un trabajo que analice los hechos que influyeron en cómo las manifestaciones y expresiones de la modernidad se concretaron tanto en la sociedad como en la arquitectura de la ciudad. Este artículo hace una revisión cronológica, entrelazando los hechos históricos del Ecuador desde lo político, lo económico y lo social, para explicar las razones de cómo se cimentaron las bases de la modernidad en la ciudad de Quito. La explicación se hace dentro de un marco de entendimiento desde las visiones de autores como Bolívar Echeverría y David Harvey, proponiendo un análisis de las particularidades históricas del país y de la ciudad. Para ello se analiza el crecimiento de la ciudad, identificando expresiones arquitectónicas representativas y evidenciando los momentos en que las bases de la modernidad se presentaron y gestaron particularidades que se manifestaron en la ciudad de Quito en respuesta a su propio modelo social, económico y político local.

\section{Palabras Claves:}

arquitectura; modernidad; Quito

\author{
Erick Santiago Camacho Aguirre ${ }^{1}$ \\ Marco Antonio Medina Ortega ${ }^{2}$
}

\begin{abstract}
The architectural texts that study modernity in the city of Quito are mostly a collection of buildings or authors and descriptions of their works, and because of this they do not contribute to an understanding of how modernity was represented into the city. Consequently, there is a lack of work that addresses the facts that influenced how the manifestations and expressions of modernity materialized both in society and the architecture of the city. This article makes a chronological review, intertwining the historical facts of Ecuador from the political, the economics and social, to explain the reasons for how the foundations of modernity were laid in the city of Quito. The explanation is made within a frame of understanding of modernity from the views of authors such as Bolívar Echeverría and David Harvey, proposing an analysis of the historical particularities of the country and the city. For this the growth of the city is analyzed, identifying the representative expressions architectural and evidencing the moments in which the foundations of modernity were presented and developed particularities that were manifested in the city of Quito in response to its own social, economic and political model.
\end{abstract}

\section{Keywords:}

architecture; modernity; Quito

\footnotetext{
${ }^{1}$ Nacionalidad: ecuatoriano; adscripción: Universidad Central del Ecuador; Doctor en Ciudad, Territorio y Sustentabilidad por la Universidad de Guadalajara, México; correo: escamacho@uce.edu.ec

${ }^{2}$ Nacionalidad: mexicano; adscripción: Universidad de Guadalajara; Doctor en Ciudad, Territorio y Sustentabilidad, por la Universidad de Guadalajara; correo: mrmedina@cucea.udg.mx
} 


\section{ANTECEDENTES}

La bibliografía existente que podría definir los períodos de la arquitectura moderna en la ciudad de Quito es limitada y no llegan a una conclusión definitiva de cómo definir a la modernidad. (Benavides Solís, 1995) (Moreira \& Álvarez, 2004) (I. Municipio del Distrito Metropolitano de Quito, 2004). Es en la actualidad que el estudio de la arquitectura moderna de Quito se ha ampliado y la han retomado investigadores de varias universidades del país. Cabe, mencionar que los esfuerzos que se están realizando están encaminados a la recopilación de información y la creación de fondos de información en trabajo conjunto con autores de reconocido renombre en la profesión.

Paralelamente, el Instituto Metropolitano de Patrimonio del Municipio de Quito, está trabajando en la identificación y posterior catalogación de bienes que serán patrimoniados y que pertenecen a lo que han llamado "Arquitectura contemporánea del siglo XX” (Ilustre Municipio del Distrito Metropolitano de Quito, s.f.). Así, se han pre identificado 125 bienes que pertenecen a este período, sin embargo, no existe una metodología clara que respalde el por qué dichos bienes fueron escogidos y forman parte del posible inventario. El actual responsable del inventario patrimonial MSc Arq. Jesús María Loor acepta que la entidad municipal no cuenta con una herramienta de análisis o reconocimiento de los valores propios de la modernidad en la arquitectura de la ciudad debido a la falta de bibliografía de respaldo por lo que han recurrido a la contratación de una consultoría que realice dicho estudio (Loor, 2017).

Entre el año 2018 y 2019 el Instituto Metropolitano de Patrimonio en cooperación con el Instituto Nacional de Patrimonio Cultural, el DOCOMOMO Ecuador y la Pontificio Universidad Católica del Ecuador han hecho varias convocatorias con el fin de socializar los criterios de valoración para la elaboración del inventario de la arquitectura moderna de Distrito Metropolitano de Quito. En su última convocatoria del 17 de septiembre de 2019 se presentó el alcance de la ficha de levantamiento de información que más allá de la información técnica, en la sección 2. Caracterización de la obra, se han identificado tres "ismos" bajo los cuales se pretende encasillar a las obras seleccionadas: Racionalismo, Funcionalismo y Organicismo. En la fase del conversatorio de dicha socialización en una de las conclusiones de los ponentes/representantes de las instituciones a cargo del trabajo de inventario se aceptó que no se cuenta con las herramientas necesarias para determinar aún la caracterización de las obras en cuestión, ni de un estudio que permita una identificación temporal de las fases de la modernidad en la ciudad.

A nivel latinoamericano los estudios de arquitectura moderna tampoco toman el caso del Ecuador como referencia. Es importante destacar que en muchos casos relevantes e históricos de la arquitectura moderna latinoamericana se evidencia la influencia de los postulados de los Congresos Internacionales de Arquitectura Moderna (CIAM) y los trabajos que Le Corbusier realizó en diferentes países como Colombia, Brasil o Argentina, en los que se pueden encontrar referencia claras a través de sus colaboradores locales ya que varios de ellos fueron colaboradores del mismo Le Corbusier como los colombianos Rogelio Salmona y Germán Samper quienes trabajaron en el Atelier 35 en París, así como el uruguayo Antonio Bonet, Oscar Niemeyer que colaboró en el diseño del edificio de las Naciones Unidas, o Amancio Williams quien colaboró en el diseño y ejecución de la Casa Curutchet en La Plata, Argentina (Maluenda, 2016).

Al analizar brevemente a los grandes exponentes de la arquitectura latinoamericana ${ }^{3}$, o al menos los más conocidos, sobresale inmediatamente la gran influencia europea en estos, ya sea porque nacieron en Europa o estudiaron y/o trabajaron en su primera etapa profesional en algún país europeo. Adicional a los ejemplos ya nombrados se puede destacar a Lina Bo Bardi de Brasil quien trabajó con Bruno Zevi o Emilio Duhart quien estudió con Walter Gropius y posteriormente, por recomendación de éste, trabajó con Le Corbusier en el proyecto de Chandigarh. Casos menos directos, pero si evidentes son las influencias de Frank Lloyd Wright o Mies van der Rohe en otros autores latinoamericanos, posiblemente debido a esta relación más estrecha con Europa. (Maluenda, 2016).

\footnotetext{
3 Ana Maluenda en su libro La arquitectura moderna en Latinoamérica hace una revisión de 18 autores latinoamericanos trascendentes e identifica que el 45\% de éstos nacieron o estudiaron en Europa, sin considerar los que trabajaron en Europa o murieron allá. Es decir que la influencia europea en la arquitectura moderna latinoamericana es evidente (La arquitectura moderna en Latinoamérica, 2016, pág. 8).
} 
Más allá de la influencia de Le Corbusier y los principios del CIAM, los estudios de la modernidad en la arquitectura en Latinoamérica se han centrado alrededor de personajes y obras de éstos, así como en su influencia en el desarrollo en la arquitectura local; sin embargo, ninguno de estos textos incluye el caso de Ecuador o de Quito. En los reconocidos estudios teóricos de Roberto Segre, Cristian Fernández Cox o Marina Waisman, entre otros, se hace énfasis en sus países de origen y se hacen análisis contextuales tomando en cuenta los grandes países latinoamericanos, la realidad es que tampoco en estos textos se analiza la situación del Ecuador.

Por otro lado, entre los trabajos que abordan el análisis de la arquitectura moderna de Quito se pueden identificar tres tipos de textos: los recopilatorios, los descriptivos y las guías. Los recopilatorios son en su mayoría realizados por el extinto Fondo de Salvamento del Municipio de Quito que conjunta diferentes autores, diferentes enfoques y carecen de un planteamiento teórico y hasta narrativo. De los documentos descriptivos se pueden identificar tres libros: Arquitectura de Quito 1915-1985 publicado por el Colegio de Arquitectos del Ecuador y Trama Ediciones de Rubén Moreira y Yadhira Álvarez; Quito 30 años de arquitectura moderna publicado por la Facultad de Arquitectura y Diseño de la Universidad Católica y Trama Ediciones ${ }^{4}$ y Arquitectura del Siglo XX en Quito de Jorge Benavides Solís publicado por el Banco Central del Ecuador. En el caso de las guías se pueden destacar dos: Ciudad de Quito guía de arquitectura del municipio de Quito y la Junta de Andalucía ${ }^{5}$ y la Guía arquitectónica de Quito de Rolando Moya y Evelia Peralta.

Después de la revisión y análisis de los textos se puede concluir que el desarrollo de éstos se basa en dos criterios: selección de arquitectos destacados (conocidos por los autores) o selección de obras destacadas. En ninguno se establece cual fue la metodología de selección de las obras o de los arquitectos que forman parte de las publicaciones, nuevamente se evidencia la ausencia de un enfoque teórico o metodológico en la selección. Queda tácitamente establecido que la importancia social de la edificación o el reconocimiento gremial o personal de sus autores es el criterio de reconocimiento para ser parte de la selección.

Es importante destacar la aproximación de Benavides Solís que pretende dejar de lado la organización cronológica o selección de autor como parte de la explicación e identificación de la modernidad en Quito y su expresión en la arquitectura de la ciudad, sin embargo, termina igualmente identificando autores y obras sin proponer una metodología o explicación del por qué las obras consideradas son relevantes o representativas.

Asimismo, es importante señalar que los textos, cuando hablan de arquitectura moderna, coinciden en ciertas fechas y establecen una franja de tiempo entre la década de los cuarenta ${ }^{6}$ a la década de los ochenta, como la etapa principal de la expresión de la modernidad en la arquitectura; en todo caso, de ninguna manera se analiza o se considera la expresión de la modernidad como objeto de estudio.

En ese contexto hay que destacar que la recuperación, la significación y la valoración del patrimonio moderno ha sido conducido por algunas iniciativas que actualmente se están llevando a cabo en ciertos países. En Holanda, por ejemplo, se creó el DOCOMOMO, organización líder a nivel mundial en conservación de la producción cultural moderna, este esfuerzo internacional evidencia la urgencia de valoración y recuperación de este legado, desafortunadamente este movimiento aún no se ha consolidado en el Ecuador, en ese sentido consideramos que la carencia de un estudio crítico y significativo de la arquitectura de esta etapa ha llevado a que no se la identifique, no se la valore y, como consecuencia, a que no se la proteja en el país.

\footnotetext{
4 El encargado de la selección de las obras que se publican tanto en el libro Arquitectura de Quito 1915-1985 como en Quito 30 años de arquitectura es Rubén Moreira (1939-2018). Aun cuando Moreira fue una de las figuras más importantes en el desarrollo del estudio de la historia y teoría de la Arquitectura del Ecuador el criterio de selección de los autores y sus obras puede cuestionarse considerando que en el libro de su autoría incluye obras personales y de otros autores que no fueron realizadas en la ciudad de Quito. (Moreira \& Álvarez, 2004, págs. $68,69,167,207)$.

${ }^{5}$ La selección de las obras de esta guía también estuvo a cargo de Rubén Moreira.

6 Tanto en Quito 30 años de arquitectura moderna, donde se incluye un relato de Sixto Durán Ballén acerca de los inicios de la arquitectura moderna de la ciudad, como en Arquitectura de Quito 1915-1985 de Moreira y Álvarez se identifican hechos coincidentes que podrían marcar el inicio de las primeras expresiones modernas en la arquitectura de Quito: la llegada del funcionalismo a las edificaciones a través de la ejecución del Plan regulador de Quito a cargo de Odriozola, Gatto Sobral, Bonino y Altamirano; la presencia de varios arquitectos europeos que se destacaron como Khon, Glass, Etwanick y Rotta y la fundación de la Escuela de Arquitectura de la Universidad Central del Ecuador, todos estos hechos en la década del cuarenta.
} 
Existen indicios de que lo anterior puede cambiar, parte de este movimiento tiene que ver con la existencia de DOCOMOMO Ecuador (DOCOMOMO, 2017) institución que en su página web expone una serie de puntos a ser analizados en una obra y expone ciertas fichas de catalogación de varias obras que han sido examinadas. La metodología expuesta responde al formato utilizado en los análisis que la organización ha elaborado en algunas partes del mundo, tomando en cuenta un amplio espectro de puntos a considerar, pero que en su mayoría son de carácter descriptivo. Destaca como punto central, de la valoración, la utilización de la tecnología del hormigón armado usado en las obras y a partir de ahí la calificación de moderna. Si bien esta tecnología es un eje fundamental comúnmente aceptado, no es suficiente al no aportar a la particularidad que significan las obras de la modernidad en la ciudad. Adicionalmente los casos presentados forman parte de ejercicios de análisis realizados en un programa de maestría local, de tal manera que si bien se sigue un procedimiento estructurado de análisis, no se expone la razón en la selección de las obras consideradas, lo que corrobora lo señalado en el presente artículo de que no existen textos de análisis arquitectónico de obras locales de este período a ningún nivel de aproximación ya sea conceptual o de representación.

De esta manera se pueden establecer varias preguntas como premisa del desarrollo del presente artículo. ¿Cómo se puede explicar conceptualmente el inicio de la modernidad y como se presentó está en la ciudad? ¿Cuáles son los elementos generales que permiten identificar a la modernidad en la arquitectura de Quito? ¿Cuáles son las bases históricas que justifican la presencia de la modernidad y cómo fue su expresión modernista?

El presente estudio parte de la propuesta de una definición de la modernidad en su ámbito más general, para el entendimiento de cuáles fueron sus momentos históricos como un marco referencial, desde un enfoque teórico que ayude a explicarla y a partir de ahí establecer cuáles fueron sus características conceptuales particulares y cómo éstas se presentaron y se expresaron en la ciudad de Quito y su arquitectura.

\section{BASES DE LA MODERNIDAD EN LA CIUDAD DE QUITO. LA PROTOMODERNIDAD}

¿Qué es la modernidad? es la premisa con la que Bolívar Echeverría intenta explicar en su famoso ensayo en el que afirma que "la modernidad es la característica determinante de un conjunto de comportamientos..." (2009, pág. 7) que, a decir de el mismo, intentan sustituir los comportamientos tradicionales de una sociedad.

De esta manera Echeverría señala:

"Tomados así, como un conjunto en el que todos ellos se complementan y fortalecen entre sí, ya de entrada estos fenómenos modernos presentan su modernidad como una tendencia civilizatoria dotada de un nuevo principio unitario de coherencia o estructuración para la vida social civilizada y para el mundo correspondiente a esa vida; de una nueva "lógica" que se encontraría en proceso de sustituir al principio organizador ancestral, y desde la que éste se percibe como obsoleto y se tolera como "tradicional"." (Echeverría, 2009, pág. 8).

Coincidente con esta explicación Sigmund Freud, analizado por Bauman en (La posmodernidad y sus descontentos, 1997), afirmaba con anterioridad que la modernidad es una cuestión de belleza, limpieza y orden, pero desde una visión unificadora que obliga al ser humano a que adopte comportamientos distintivos que ponen en cuestión a los tradicionales. Echeverría amplía su explicación con una descripción de las características de la modernidad en tres puntos principales: la técnica científica, la secularización de lo político y el individualismo, aspectos que se abordaran más adelante.

Por su parte Echeverría (2009) afirma que la modernidad está ligada al proceso de mestizaje, aspecto en el que coincide con Cristian Fernández Cox (1991), es decir que los primeras manifestaciones modernistas se producen posterior a la conquista, cuando Latinoamérica sufre la imposición cultural por parte de los países europeos, principalmente de España.

Tiene sentido esa afirmación ya que en principio los pueblos indígenas americanos fueron sometidos a un proceso de unificación que abarcó todos los niveles de su estructura social, política y económica, sin considerar las condiciones existentes. En su libro América Latina en su arquitectura Roberto 
Segre (1975) cita a Fernando Chueca afirmando a la arquitectura como uno de los tres grandes legados que se heredó de España en el proceso de la colonia. Sin embargo, es importante destacar que no todo lo producido desde la época de la conquista debe ser entendido como expresión moderna, al menos no en el caso de la Arquitectura ya que la afirmación compartida de Echeverría y Fernández Cox se refiere al proceso mismo de modernidad como estructura de pensamiento, más no como expresión modernista.

De acuerdo con (Treviño Moreno, 2000) el pensamiento moderno podría dividirse en tres etapas, tomando como principio los sucesos desde el renacimiento, que en el caso de Latinoamérica están marcados por la conquista como lo afirman Echeverría y Fernández Cox. La primera etapa que empieza con el rompimiento del pensamiento medieval marcando grandes cambios sobre todo en lo humanístico con la introducción de los textos de la cultura griega y latina; dentro de esta primera etapa, de más de un siglo de duración, se debe mencionar los cambios políticos producidos por las propuestas reformistas de Lutero que marcan el inicio de la independencia, al menos en Europa, de la hegemonía del catolicismo. Tal vez el impacto más importante del pensamiento fue en lo científico, aunque demoró en materializarse, pero se cambia el modelo puramente metafísico dando paso a un mundo regido por la químicafísica-biología, en lo cultural destaca la etapa de la conquista y colonización de otros continentes por parte de las monarquías europeas, dando lugar a la consolidación de estados monárquicos de la mano de la explotación de las materias primas de los territorios conquistados.

En la segunda etapa se consolida el pensamiento racional de la mano de los postulados de René Descartes (1586-1650) y otros filósofos, estableciéndose lo que conocemos como la ilustración. En este surgimiento se profundiza el método científico que dio paso al aporte de otros grandes pensadores como Spinoza o Leibnitz, y llegando a su punto más alto un siglo después con los aportes al conocimiento de Isaac Newton. Sin embargo, es con Kant que el pensamiento empírico y el racional encuentran un punto de encuentro unificando el pensamiento occidental que a partir de este punto se podría decir que se ha consolidado una nueva cultura de la modernidad de acuerdo con (Treviño Moreno, 2000). En lo político se consolido el estado monárquico absoluto a partir del vínculo entre la aristocracia y la iglesia, hecho que se profundizó en los territorios conquistados especialmente en América. Este progreso del conocimiento racional se extiende por todos los territorios dando paso por un lado a la extensión del conocimiento en todos sus niveles, constituyéndose en la cimentación de la etapa industrial, y por otro a la secularización de la sociedad poniendo como centro del pensamiento a la razón por sobre la fe (Treviño Moreno, 2000) que fue la base del pensamiento utópico propio de la ilustración.

La tercera etapa está marcada por varios hechos que determinaron el nacimiento del estado moderno con fundamento en los pensamientos de Hegel y Marx y que dieron paso a las grandes revoluciones sociales, particularmente la francesa que marca el inicio del fin de las monarquías y la separación de la iglesia de los estados. Aun cuando varias monarquías europeas se reestablecieron durante el siglo XIX, la base de pensamiento en la que se asentaban estaba ya desgastado y terminaron de extinguirse hacia la Primera Guerra Mundial con la revolución bolchevique (Treviño Moreno, 2000). Es así como el aparato industrial europeo alimentado por la explotación de recursos primarios de los territorios conquistados consolida el modelo del capital y de poder burgués a lo largo de todo el siglo XIX y que provocó el crecimiento de las grandes urbes que nacieron precisamente en el cambio de modelo de intercambio entre el campo y la urbe. Esto llevó al surgimiento de nuevas necesidades sociales y de infraestructura, que es en donde nace la ciudad industrial (Aymonino, 1972), se establece de esta manera el pensamiento de progreso dentro de la maquinaria social soportadas principalmente en la ciencia y la tecnología (Treviño Moreno, 2000).

Ya en términos de expresión modernista como tal David Harvey (1990) hace un análisis de la modernidad, toma como base lo expresado por Charles Baudelaire quien la definió en 1863 como: "la modernidad es lo efímero, lo veloz, lo contingente; es una de las mitades el arte, mientras que la otra es lo eterno y lo inmutable" (Harvey, 1990, pág. 25). Para Harvey el concepto de la destrucción creadora es fundamental para el entendimiento de la modernidad, desde los principios establecidos por Nietzsche hasta las ideas desarrolladas por Schumpeter, en donde la figura del capital como motor de los hechos económicos y sociales son la base fundamental de la producción cultural. 
"Por lo tanto, es importante tener en cuenta que el modernismo que apareció antes de la Primera Guerra Mundial fue más una reacción a las nuevas condiciones de producción (la máquina, la fábrica, la urbanización), circulación (los nuevos sistemas de transporte y comunicaciones) y consumo (el auge de los mercados masivos, la publicidad y la moda masiva) que un pionero en la producción de esos cambios.” (Harvey, 1990, pág. 39).

De esta manera se identifica a la urbanización (y por consiguiente todos sus componentes entre ellos los objetos arquitectónicos) como una de las manifestaciones de la modernidad en respuesta a los nuevos modos de producción ${ }^{7}$ (Aymonino, 1972), resulta imprescindible identificar cuando estos cambios se produjeron en el Ecuador como premisa de la modernización de la ciudad de Quito $\mathrm{y}$, por tanto, identificar las primeras manifestaciones modernas. Está claro que estas influencias y transferencias se produjeron en el país siempre de manera tardía, siempre en respuesta natural a la velocidad en que la información, tecnología y el conocimiento mismo llegó al territorio ecuatoriano.

Así, se pueden identificar dos momentos importantes en los orígenes de la modernidad en el país, primero la caída del estado oligárquico terrateniente a finales del siglo XIX como consecuencia de las políticas que procuraron la modernización del estado ecuatoriano promulgadas por el presidente Gabriel García Moreno que por cierto se establecieron en un contexto de contradicción de principios que terminó provocando la etapa liberal ya entrando al siglo XX. Como lo narra Enrique Ayala Mora en su Resumen de la historia del Ecuador:" El programa garciano descansó sobre una contradicción. Por una parte, impulsó la modernización y consolidación estatal, estimuló la producción y el comercio, desarrolló la ciencia y la educación; por otra, impuso una ideología reaccionaria excluyente y represiva, con la dictadura clerical terrateniente". (Ayala Mora, 2008, pág. 30).

Ayala Mora (2008) destaca que una de las inversiones más importantes realizadas por el plan de García Moreno fue en el campo de la educación, así se crearon entre otros la Escuela Politécnica Nacional, el Observatorio Astronómico, el Colegio San Gabriel y el Teatro Sucre (ver tabla 1 p. 113).

Para la concepción, planificación y posterior construcción de todas estas edificaciones había una gran carencia de profesionales nacionales que puedan enfrentar dicho encargo de tal manera que se recurrió a expertos extranjeros tales como Antonio Russo (1889-1967), Lorenzo Durini (1955-1909) y Francisco Durini (1880-1970) todos de origen italiano y Pedro Brüning (1886-1936) de origen alemán. En el caso de figura locales relevantes como Pedro Aulestia (1881-?) o más tarde Luis Felipe Donoso (1899-1977) se formaron en Italia y Francia respectivamente (del Pino, 1993).

La etapa garciana resultó fundamental para el desarrollo socio-político del estado ecuatoriano, tanto como un proyecto de consolidación de la estructura del estado como reactivo fundamental para la etapa de la revolución liberal de fines del siglo XIX e inicios del siglo XX, posteriormente bajo la presidencia de Eloy Alfaro se consolida el modelo económico agroexportador, impulsado por la producción en la costa de los exportadores de cacao que juntaron su poder económico alrededor de Guayaquil. Si bien este modelo mantuvo y reafirmó el regionalismo que además sectorizó los diferentes productos, también logró articular un proyecto unificador de país que Ayala Mora lo denomina el "proyecto nacional mestizo". (Ayala Mora, 2008, pág. 32).

Es en esta etapa de la revolución liberal (1895-1912) en la que se producen los cambios más profundos, resultando oportuno retomar lo propuesto por Harvey (1990) respecto de que el modernismo previo a la Primera Guerra Mundial fue consecuencia de nuevas formas de producción, circulación y consumo. Dentro de los principales cambios en el Ecuador encontramos que el ferrocarril permitió comunicar la sierra con la costa, transformándose en principal medio de transporte nacional, se consolida la producción agrícola como motor fundamental de la economía y se complementa con el surgimiento de una creciente clase productiva dedicada a la comercialización de la gran producción agrícola,

\footnotetext{
7 En su libro Orígenes y desarrollo de la ciudad moderna (1972) C. Aymonino hace un análisis de la ciudad industrial y establece como una de las principales razones de su surgimiento a la disolución de las antiguas relaciones económicas de propiedad de uso del suelo provocado principalmente por la influencia de la industria en el campo más que en la urbe, sobre todo por la modernización de las actividades agrícolas, si bien este efecto no fue el mismo en todos los países, si provoco nuevos intercambios comerciales en todos y en todas las urbes.
} 
modificando las formas de consumo de la sociedad. Por tanto, se puede afirmar que el inicio del estado moderno empieza con las políticas de García Moreno en la segunda mitad del siglo XIX y termina consolidándose y materializándose en el proyecto liberal de inicios del Siglo XX.

Tabla 1. Extracto de obras realizadas en la ciudad de Quito entre los años 1820-1922

\begin{tabular}{|c|c|c|}
\hline Años & Obra & Constructor \\
\hline $1832-1866$ & Casa de la Moneda & \\
\hline 1838 & Colegio Militar & \\
\hline 1847 & Palacio de Gobierno & \\
\hline $1869-1875$ & Hospicio & F. Schmidt \\
\hline $1869-1875$ & Panóptico & Thomas Reed \\
\hline 1870 & Escuela Politécnica Nacional & Padre Menten \\
\hline 1872 & Colegio San Gabriel & \\
\hline $1873-1892$ & Observatorio Astronómico & P. Menten, Dressel \\
\hline $1880-1887$ & Teatro Sucre & F. Schmidt \\
\hline $1884-1910$ & Escuela de Artes y Oficios & F. Schmidt \\
\hline 1893 & Seminario Mayor & F. Schmidt \\
\hline $1895-1897$ & Alumbrado público & \\
\hline 1897 & Inicio construcción ferrocarril Quito-Gye & Arcehr Harman \\
\hline $1897-1904$ & Mercado de Santa Clara & F. Schmidt y Pérez \\
\hline 1897 & Colegio Mejía & \\
\hline 1899 & Maternidad & F. Schmidt \\
\hline 1900 & Conservatorio Nacional de Música & \\
\hline 1900-1913 & Hospital Militar & F. Schmidt \\
\hline 1901-1924 & Instituto Normal Juan Montalvo & \\
\hline $1908-1920$ & Estación del Ferrocarril Quito & P. Aulestia \\
\hline 1914 & Liceo Fernández Madrid & P. Aulestia \\
\hline 1914-1924 & Edificio de los Correos & Ag. Ridder \\
\hline 1914 & Normal Manuela Cañizares & E. Adler \\
\hline 1914 & Tranvías eléctricos & \\
\hline 1915 & Escuela Municipal Espejo & P. Aulestia \\
\hline 1917 & Teatro Variedades & \\
\hline 1917-1936 & Círculo Militar & \\
\hline & Biblioteca Nacional & F. Durini \\
\hline 1922 & Escuela de Bellas Artes & P. Aulestia \\
\hline 1922 & Escuela 24 de mayo & \\
\hline
\end{tabular}

Fuente: Tomada de Del Pino, 1993

\section{LA DIMENSIÓN TÉCNICA CIENTÍFICA COMO BASE DE LA MODERNIDAD EN LA CIUDAD DE QUITO}

Ahora, tomando como base las características de la modernidad como las define Echeverría (2009) el primer punto es el de la técnica científica en donde resalta la confianza del ser humano en el uso de la razón como base de pensamiento dejando de lado a la metafísica, a partir de ahí se empiezan a desarrollar todo lo medible tanto de la naturaleza como del mundo social; de esta manera confirma a la ciudad como base fundamental del hombre moderno y en donde se desarrolla precisamente el progreso técnico.

Para el caso del Ecuador esta fase se constituye durante el período en que se forman varias empresas que aportan al desarrollo de la sociedad en todos los niveles, se destaca la actividad de telecomunicaciones, la empresa de ferrocarriles 
que se inaugura hacia 1908 y que termina con el aislamiento de Quito, se crea la empresa Cemento Nacional, alcantarillado, agua potable y luz eléctrica (Ver Tabla 1). De esta manera se responde a la creciente migración interna que principalmente se produjo hacia las tres grandes ciudades del Ecuador: Quito, Guayaquil y Cuenca. Hay que destacar que el crecimiento de la ciudad de Quito fue más lento en comparación con Guayaquil que se transformó en el principal polo económico del país.

Como se puede apreciar en los mapas de Quito de 1883 (ver Imagen 1) y de 1903 (ver Imagen 2 p. 115) el crecimiento no es realmente notorio. Guayaquil superaba los 70.000 habitantes mientras Quito superaba los 50.000 (I. Municipio del Distrito
Metropolitano de Quito, 2004). El crecimiento de la ciudad fue sostenido hasta los años 20 , pero es a partir de la consolidación del proyecto liberal que se nota el crecimiento de la ciudad. Esta etapa está marcada por el control político de las oligarquías liberales y el crecimiento del aparato burocrático.

No olvidar que el crecimiento urbano de la ciudad de Guayaquil fue antes que el de Quito, pero también la aplicación de avances tecnológicos en las construcciones. Ya en el año 1908 se inaugura el Mercado $\mathrm{Sur}^{8}$ (actual Palacio de Cristal) en Guayaquil, que es una estructura completa de acero cuyas piezas fueron importadas de Bélgica (Empresa Pública Municipal de Turismo, Promoción Cívica y Relaciones Internacionales de Guayaquil, EP., 2018).

Imagen 1. Mapa de Quito, 1883

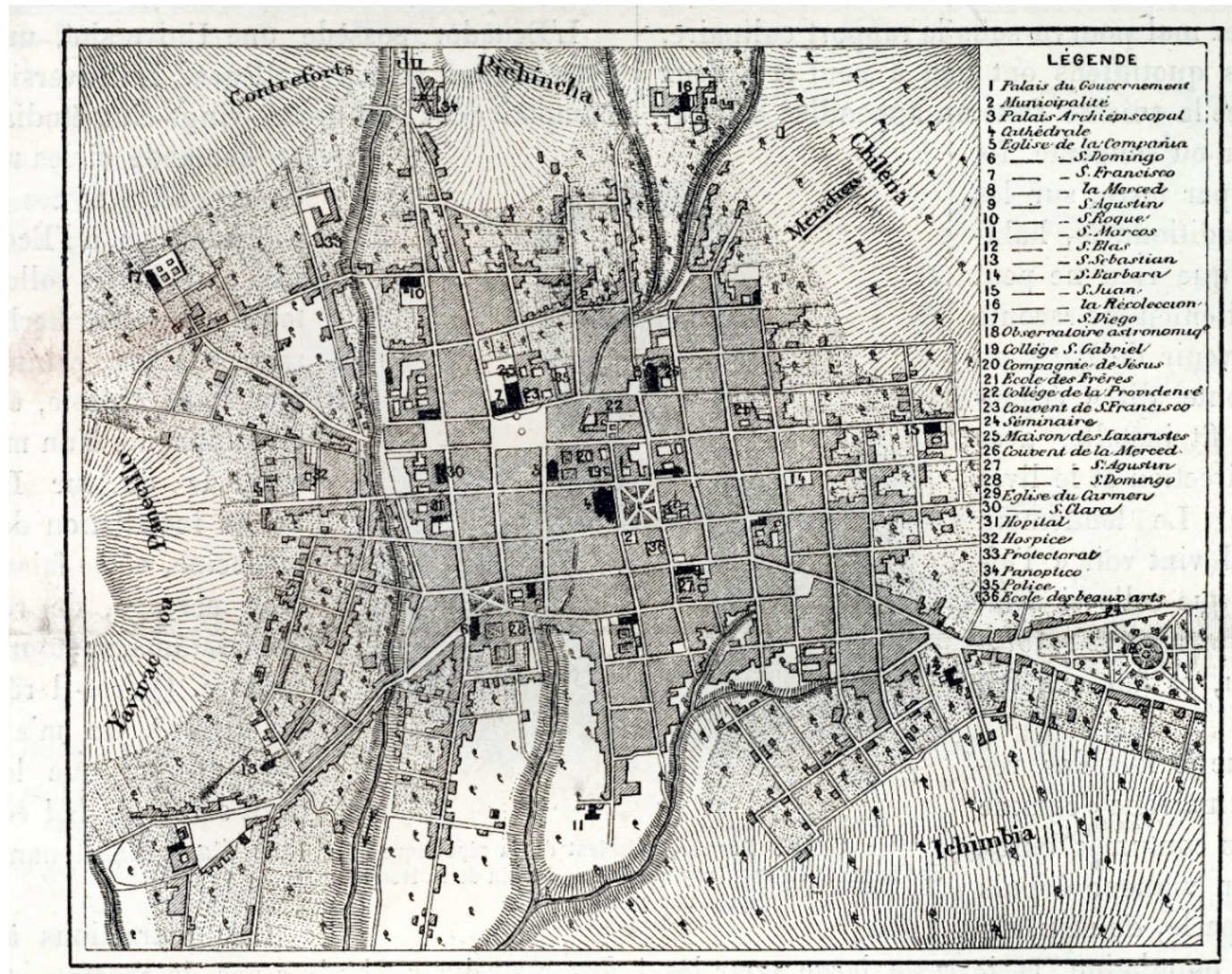

Fuente: Archivo histórico del Distrito Metropolitano de Quito

\footnotetext{
8 El Antiguo Mercado Sur, según consta en la información proporcionada por la página web oficial de la municipalidad de Guayaquil "fue diseñado y construido por los Ingenieros Francisco Manrique Pacanisy Carlos Van Ischot. Las piezas utilizadas para su construcción fueron traídas directamente desde Bruselas, Bélgica y fue prefabricado por la firma Verhaeren Ca JagerIngeneriusConstruiters de Bruxelles". (Empresa Pública Municipal de Turismo, Promoción Cívica y Relaciones Internacionales de Guayaquil, EP., 2018) En el año 2002 el edificio fue relocalizado en predios del conocido Malecón 2000 y funciona como centro de exposiciones conocido como el Palacio de Cristal.
} 


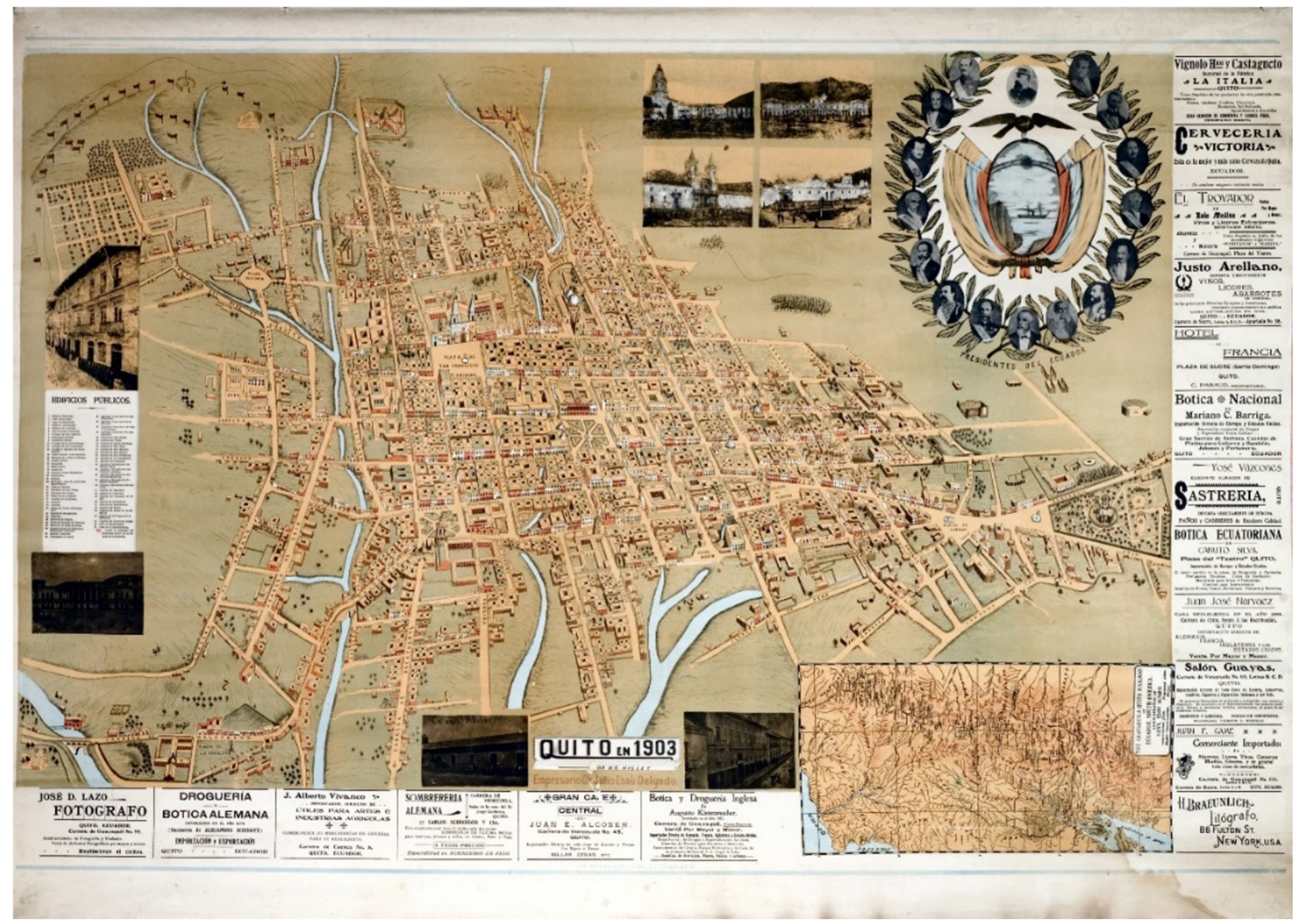

Fuente: Archivo histórico del Distrito Metropolitano de Quito

Para 1905 se termina de construir el edificio de la Cárcel Municipal de Guayaquil ${ }^{9}$ que es el primer edificio concebido y construido con hormigón armado. Si bien en sí el edificio es de estructura mixta (ladrillo, piedra, madera y hormigón), es el primero del que se tiene registro en el país en el que se haya utilizado hormigón armado en losas y columnas. No está por demás señalar que los materiales utilizados fueron importados desde Italia, introduciendo esta técnica innovadora para la época y que en el caso de la ciudad de Quito se introdujo muchos años después (Empresa Pública Municipal de Turismo, Promoción Cívica y Relaciones Internacionales de Guayaquil, EP., 2018).

Posteriormente el crecimiento de la urbe costeña se vio truncada con la crisis de la industria del cacao, que era la base económica de la región, debido a sendas plagas que afectaron las plantaciones y la crisis de posguerra de los países europeos, lo que resultó en un creciente deterioro de las condiciones económicas que fueron principalmente trasladadas a los trabajadores. De esta manera se produce una revuelta de la clase trabajadora de la costa que culmina con la matanza conocida como el "bautizo de sangre" en las calles de Guayaquil (I. Municipio del Distrito Metropolitano de Quito, 2004).

La crisis del crecimiento económico en la costa tuvo un efecto en Quito como urbe que empezó a consolidar su dinámica económica alrededor de su condición de centro político, además de la influencia de la conexión comercial que provocó el ferrocarril cuya estación ya aparece en el mapa de la ciudad de 1914 (ver Imagen 3 p. 116), asimismo, el trazado de la ciudad empieza a trascender el parque de la Alameda hacia el norte.

\footnotetext{
${ }^{9}$ Este edificio fue construido por el arquitecto venezolano Francisco Manrique Pacanis. El edificio fue inaugurado en el año 1907 y funcionó como centro de detención hasta el año 1950 cuando cerró sus puertas. Actualmente, el edificio se encuentra abandonado a la espera de su intervención para albergar un Centro de Desarrollo Cultural, proyecto realizado por la Empresa Arquitectura Urbana en el año 2017.
} 


\section{Imagen 3. Mapa de Quito, 1914}

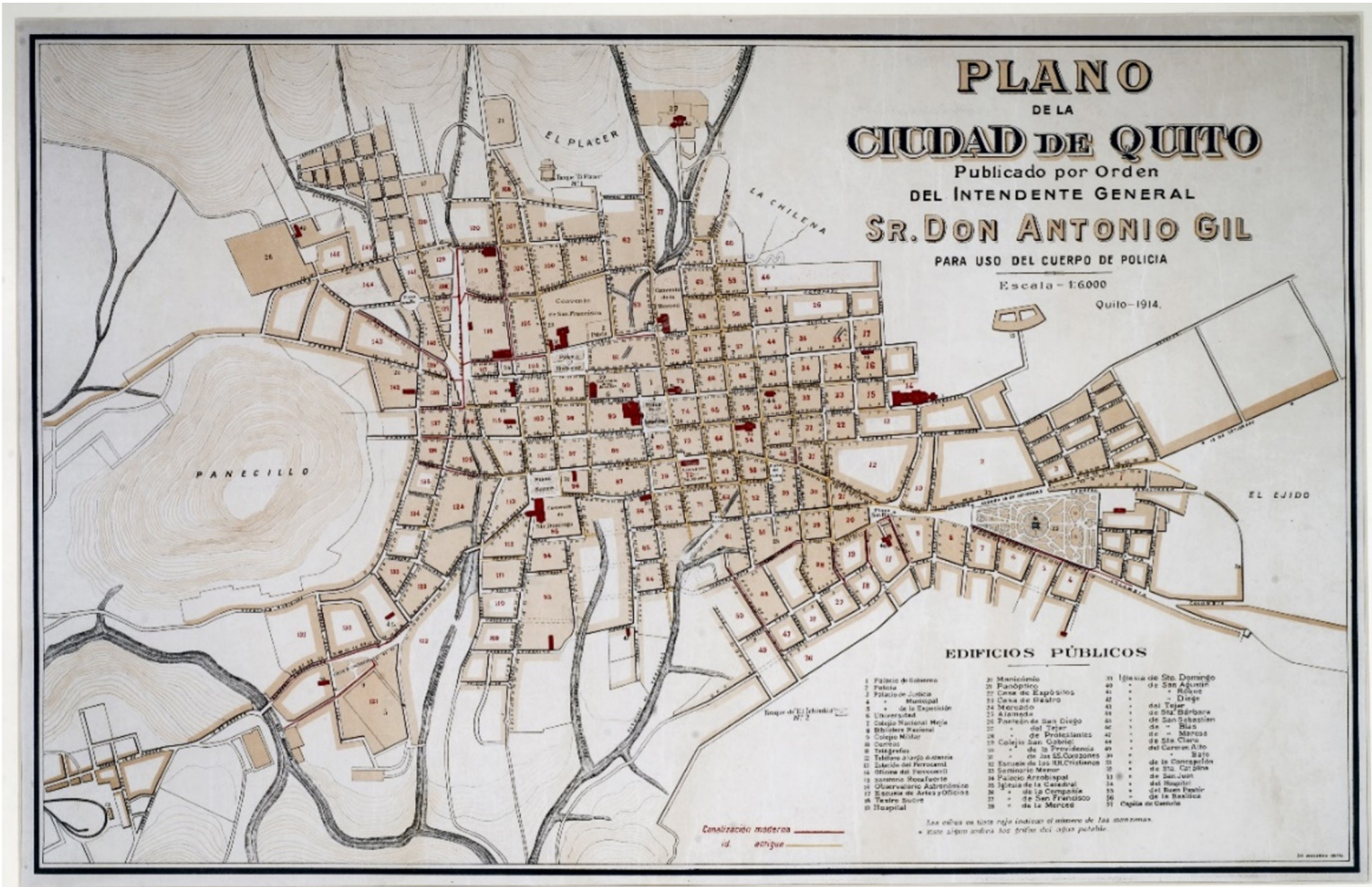

Fuente: Archivo histórico del Distrito Metropolitano de Quito

Carlos Maldonado ${ }^{10}$ (La arquitectura de Quito en la época republicana, 1993) describe esta etapa de la siguiente manera:

"La construcción del ferrocarril de Guayaquil a Quito, terminado en 1909, determinó un aumento notable del comercio entre la sierra y la costa y una concentración mayor de la población en los centros urbanos servidos por dicho ferrocarril. Especialmente se aceleró el crecimiento de las dos ciudades terminales de la vía, es decir, Quito y Guayaquil. En Quito, las antiguas residencias coloniales comenzaron a convertirse en casas de alquiler y las familias acomodadas empezaron a establecerse en nuevos barrios trazados hacia el norte de la ciudad. En estos barrios se empezaron a construir residencias unifamiliares rodeadas de jardines imitando los "barrios-jardines" de Inglaterra y las casas campesinas o "chalets" de Suiza y Francia; pero dentro de las técnicas constructivas tradicionales del país." (Maldonado, 1993, pág. 146).

Como se puede ver el mapa de Quito del 24 de mayo de 1922 (ver Imagen 4 p. 117) ya se identifican el barrio La Magdalena al sur del Panecillo, el barrio América y el barrio La Mariscal hacia el norte, tal como lo describe Maldonado. De esta manera, se evidencia la influencia del ferrocarril como generador de las nuevas dinámicas comerciales y de comunicación en una ciudad que demoró décadas en crecer y que pasa a casi duplicar su superficie planificada en menos de diez años.

\footnotetext{
10 Maldonado describe brevemente sobre la crisis de la arquitectura tradicional principalmente causada por la influencia internacional que viene introduciendo nuevas tecnologías y que aún no encuentra una expresión particular, más bien interviene en los modelos tradicionales que en la época se encontraban revisando los modelos clásicos pero desarrollados con nuevos materiales insertados en modelos constructivos tradicionales.
} 


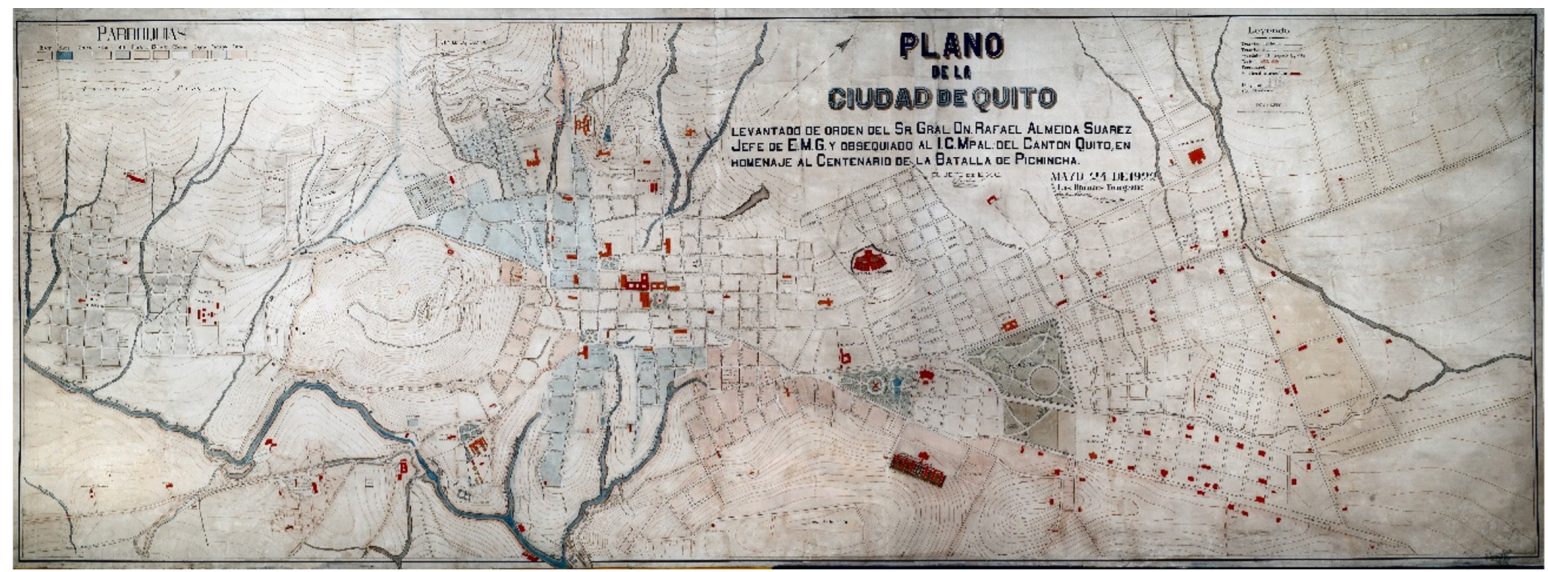

Fuente: Archivo histórico del Distrito Metropolitano de Quito

A manera de resumen Inés del Pino (1993) hace un registro de las edificaciones más importantes construidos en la ciudad desde la época republicana hasta 1922 donde se puede evidenciar claramente como desde la época garciana (1869-1893) se empieza a invertir en la infraestructura estatal, educativa, científica y religiosa, característica del modelo, en contraste y en respuesta se ve como la inversión en el área del desarrollo social en la época de la revolución liberal (1895-1912) se expresa en las edificaciones de comunicación, educación laica y otras edificaciones de desarrollo cultural, expansión de la ciencias e infraestructura y que se pueden apreciar en la tabla 1.

No podemos pasar por alto que en la lista elaborada por Inés del Pino no se identifica una de las edificaciones emblemáticas de la época como es el Mercado de Santa Clara ${ }^{11}$ diseñado por Francisco Schmidt y Gualberto Pérez. La estructura de este edificio fue importada desde Hamburgo, Alemania en el año 1899 y su construcción duró hasta el año 1904 cuando fue inaugurado. Es importante hacer esta aclaración ya que el Mercado de Santa Clara fue la primera construcción en estructura de acero construida en la ciudad (Bienal de Arquitectura de Quito, 2018). Asimismo, es importante señalar al Pasaje Royal como una de las primeras manifestaciones de cambio morfológico en las construcciones, ubicado en el Centro Histórico, el edificio fue concebido para oficinas y comercio y no para vivienda (Benavides Solís, 1995), además de ampliar las aperturas en planta baja con el fin de albergar las primeras vitrinas comerciales como una influencia directa de los pasajes comerciales de Europa, tal como las describía Walter Benjamin en su libro de los pasajes (ver Imagen 5).

\section{Imagen 5. Pasaje Royal (tomada entre 1915-1925)}

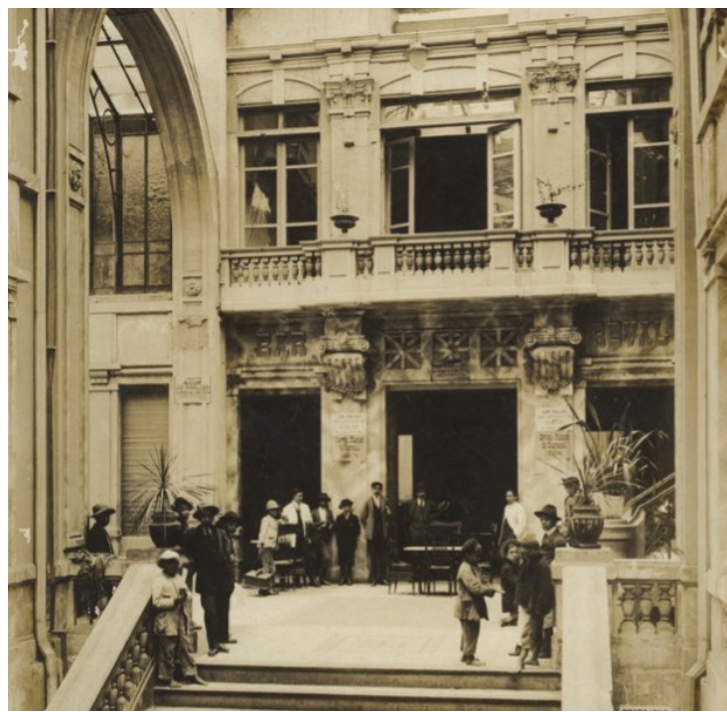

Fuente: Autor desconocido, Instituto Nacional de Patrimonio Cultural, 2015

\footnotetext{
${ }^{11}$ El mercado funcionó hasta el año 1980 hasta que fue desarmado y se lo reconstruyó en el Parque Itchimbía para albergar el actual Centro Cultural Itchimbía conocido como el Palacio de Cristal. Tanto el Mercado Sur de Guayaquil como el de Santa Clara de Quito fueron obras del gobierno liberal e introducen la construcción en acero como expresión importada desde el extranjero. Las similitudes en su reutilización son evidentes y fueron realizadas en épocas similares sin embargo creemos que son más una coincidencia conceptual más no política ya que la relación entre las administraciones municipales de las dos ciudades no existe, son más bien independientes.
} 
En la lista tampoco aparece el Palacio de la Exposición Nacional de 1909, actual Ministerio de Defensa, este edificio tiene relevancia por un lado por el uso del hormigón armado en las columnas que sostenían la cúpula de acceso ${ }^{12}$, y por otro lado por su ubicación en la Recoleta, sitio ubicado entre el centro de la ciudad y la estación del ferrocarril de Chimbacalle, dando cuenta como la proyección de la llegada del ferrocarril afectó al crecimiento de la ciudad.

Como se evidencia, los efectos del desarrollo tecnológico en la sociedad quiteña se vieron marcada por la construcción y funcionamiento del ferrocarril como motor del modelo de intercambio comercial y que en primera instancia afectó territorialmente en el crecimiento abrupto de la ciudad a partir de este momento. Sin embargo, este cambio tecnológico no afectó directamente a la arquitectura que, como lo dice Maldonado (1993), más bien tuvo efectos en su conformación morfológica más no es su técnica constructiva. En tal caso, la inclusión de baterías sanitarias y sistemas hidráulicos en las edificaciones son los cambios más importantes en las construcciones desde la época de la colonia, sin que esto implique un cambio en la manera tradicional de edificar en la ciudad. El cambio morfológico se produjo una vez que la ciudad trascendió los límites del Centro Histórico y, sobre todo, en el barrio La Mariscal donde se implantó el modelo de ciudad jardín con retiros aislando las edificaciones (Maldonado, 1993).

\section{LA DIMENSIÓN SECULARIZACIÓN DE LO POLÍTICO COMO BASE DE LA MODERNIDAD EN LA CIUDAD DE QUITO}

En segundo término, Echeverría define a la modernidad como la secularización de lo político, en ese contexto consideramos que la transición del modelo garciano al liberal también evidencia este cambio a través de la proclamación del estado del Estado Laico. Las evidencias más claras y simbólicas de este contraste político se pueden ver en la creación y construcción del Colegio Nacional San Gabriel en la época garciana y, posteriormente, la construcción del Colegio Nacional Mejía que se considera como la primera institución nacional laica. Sin embargo, esta declaración del estado laico y separación con la iglesia como parte del Estado, no eliminó la hegemonía del pensamiento católico sobre la cultura y sociedad quiteña.

Si bien la construcción de colegios nacionales laicos aumentó y multiplicó, también se fundaron instituciones educativas privadas de origen católico que captaron población con recursos económicos y con ello reconcentraron y siguieron formando parte de la sociedad que detenta el poder entre sus manos. Es decir, el Estado separo a la iglesia católica, pero esta no perdió su poder en la sociedad, en ese contexto la clase social media ligada al comercio se fortaleció en el ámbito de la política. En lo económico llegó una incipiente industrialización, pero la agroexportación siguió siendo la base económica del país, principalmente del cacao y marginalmente la producción de arroz, café y caña de azúcar. (Ayala Mora, 2008).

A partir de 1925 con el derrocamiento del presidente Cordero por parte de los militares, empieza una nueva etapa de alrededor de dos décadas que, coincidente con la crisis mundial del modelo capitalista de la primera posguerra, llevó a que el país no supere su modelo agroexportador, más bien se profundizó en el mismo, pero con una clara diversificación de productos lo que hizo que su modelo sea cada vez más dependiente de grupos monopólicos que acapararon la producción nacional.

En ese contexto el Estado Ecuatoriano siguió consolidándose en su institucionalización, prueba de esto es la creación del Banco Central por parte del presidente Isidro Ayora en el año 1928. (Ayala Mora, 2008). De esta manera se fortalecieron los sectores intermedios de comercio, la burocracia estatal y la banca, impulsando el crecimiento urbano de Quito como se puede ver en el mapa de 1931 (ver Imagen 6 p. 119), para ese entonces es por demás evidente que el trazado trascendió los límites de la calle Colón en cuyos límites se asentaron principalmente familias acaudaladas con intereses en los sectores de la industria, comercio y la política, entre otros (Benavides Solís, 1995).

Esta etapa entreguerras y la crisis mundial está marcada por un cambio de pensamiento en

\footnotetext{
12 Mauricio Luzuriaga en su capítulo Los Arquicrímenes de Quito del libro Arquitectura de Quito una visión histórica, toma una crónica de El Comercio que dice: "En el centro, en primer término, se levanta el Pabellón ecuatoriano. Construido de dos pisos, sus columnas de cemento armado soportan la artística armadura, que corona la cúpula de treinta metros de altura...” (1993, pág. 180)
} 
la búsqueda de nuevos lenguajes de expresión en todas las esferas del arte y el de la arquitectura no es la excepción. En el año de 1928 se funda el CIAM (Congreso Internacional de Arquitectura Moderna) a través del cual se pretendía hacer frente a los nuevos desafíos que la ciudad debió encarar producto de la revolución industrial y las necesidades de los tiempos modernos, sin embargo, estos principios tendrán efecto en la ciudad más adelante.

\section{LA DIMENSIÓN INDIVIDUALISMO COMO BASE DE LA MODERNIDAD EN LA CIUDAD DE QUITO}

En un tercer término, Echeverría caracteriza a la modernidad a través del individualismo, entendido como un comportamiento social práctico que presupone que el motor de la realidad humana es el individualismo social y el colectivo. En la etapa posliberal se sentaron las bases de los movimientos sociales y la estructuración del estado y del bienestar social con el pensamiento liberal. Si bien el poder de estado se mantuvo en los grupos monopólicos, se emitieron leyes que buscaban encontrar un equilibrio a favor de la fuerza de trabajo. Así en el año 1937 Alberto Enríquez Gallo emite el Código del Trabajo, entre otras medidas nacionalistas (Ayala Mora, 2008).

Imagen 6. Mapa de Quito, 1931
El caso de la arquitectura de Quito de este período tuvo una expresión ecléctica muy marcada por las nuevas tecnologías como el hormigón y el acero, ya muy utilizados en los países europeos, pero que no lograban establecerse como técnica constructiva primordial, más bien empezaron a introducirse en las edificaciones a través de la construcción de dinteles (González, Sosa \& Merino, 1993). Por otro lado, los profesionales extranjeros acaparaban los trabajos que principalmente eran financiados por las personas acaudaladas. Incluso llegaron a ser reconocidos a nivel nacional con la "Orden Nacional al Mérito", ejemplo de lo anterior son los arquitectos Vinci y Durini.

Las referencias y formación de quienes ejercían la profesión en esta época no ejercieron un impacto a nivel de la técnica y la tecnología, más bien fueron imposiciones formales y decorativas que llegaron a romper con la continuidad que había iniciado desde la llegada de los españoles y que había alcanzado ciertos rasgos particulares, que si bien se pueden considerar mestizos, hacían muy clara la diferenciación cultural (Benavides Solís, 1995) para el caso de Quito. Es recién hacia finales de la década de los treinta que aparece en Quito el Hotel Humboldt que se conoce como uno de los primeros edificios de hormigón armado, diseñado en Estados Unidos por la empresa Hopkins y Dentz y el ingeniero Alberto Mena Caamaño. El edificio se terminó de construir en el año 1939 (I. Municipio del Distrito Metropolitano de Quito, 2004). ${ }^{13}$

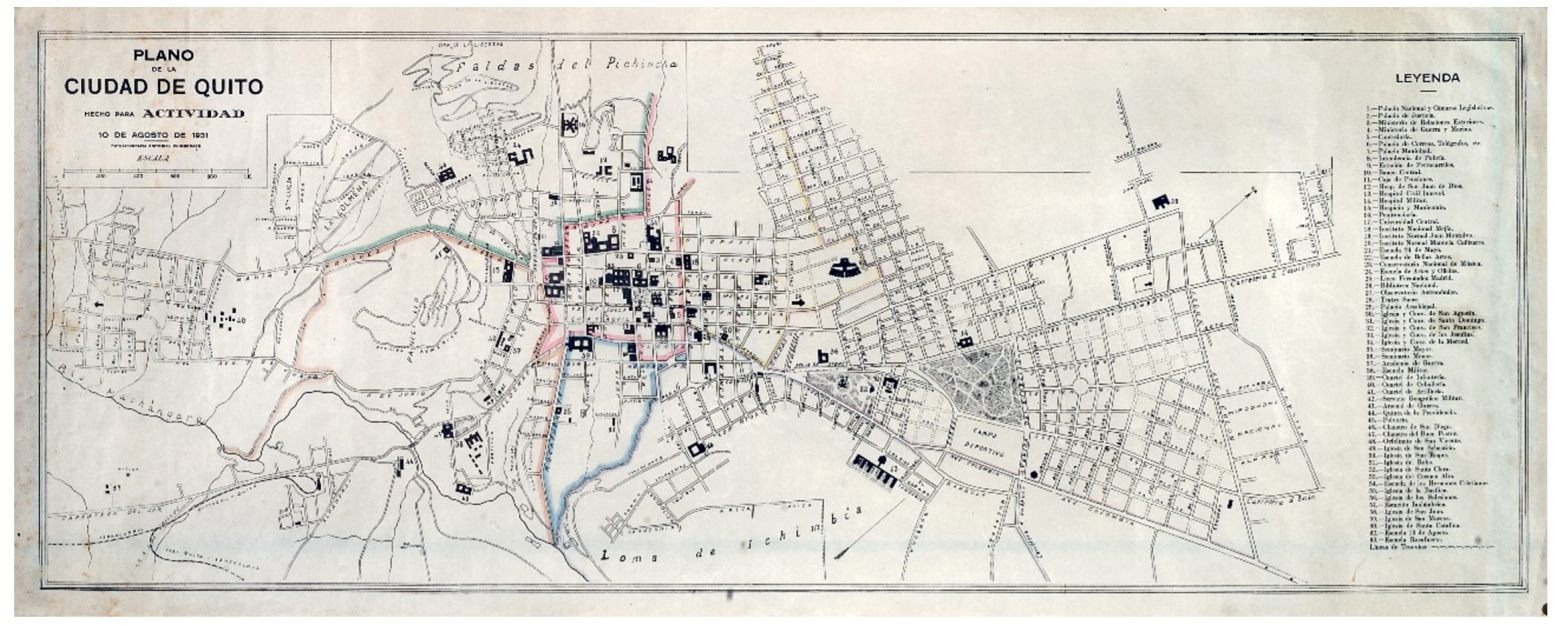

Fuente: Archivo histórico del Distrito Metropolitano de Quito

\footnotetext{
13 Este edificio es conocido como el Banco la Previsora, ya que albergó a este una vez que dejó de funcionar como hotel. No existe un estudio que determine cual es el primer edificio hecho de hormigón armado en la ciudad, sin embargo, se identifica al Hotel Humboldt como uno de los dos como los primeros de los que se tiene registro. El otro edificio es la Antigua Caja de Pensiones (Maldonado, 1993)
} 
Mies van der Rohe en su artículo Arquitectura y modernidad, publicado en $1924^{14}$ aseguraba que las edificaciones son impersonales por naturaleza, y que más bien son el símbolo de una época. De esta manera justificaba la inutilidad del uso de las formas del pasado reivindicando el pensamiento laico de esos tiempos. Pues evidentemente la arquitectura, una vez que empezó a aportar como expresión de la modernidad hizo exactamente lo contrario a lo postulado por Mies. Es precisamente a través de las individualidades que la modernidad empezó a expresarse en arquitectura. Así lo demuestra la estructura del Congreso Internacional de Arquitectura Moderna (CIAM) que basó sus postulados y su difusión en sus figuras rutilantes. Al igual que la búsqueda de las vanguardias en las artes que nació a principios de siglo, la arquitectura, entre posguerras "se ve envuelta en esta vorágine y experimenta el vértigo de la superación, experimenta la totalización estética; padece y produce una fascinación vanguardista, pero se ve siempre obligada a medirse con su propia e inevitable pragmaticidad." (Masiero, 2003, pág. 214).

\section{CONCLUSIONES}

El presente trabajo se construye bajo dos líneas principales: una visión general de la modernidad propuesta por Echeverría y otra con énfasis en las particularidades que proviene de D. Harvey acerca de la expresión del modernismo como materialización de la modernidad misma. Los procesos de la modernidad y modernización como sociedad ecuatoriana empezaron en el país con el proyecto planteado por el entonces presidente Gabriel García Moreno (1821-1875), que en el documento se hace alusión como etapa garciana, periodo en que se emiten los primeros planteamientos unificadores a nivel social y político en Ecuador. Sin embargo, este proceso fue propio de una dinámica social y económica de la época que transcurrió lentamente en nuestro país, pero que además se acompañó por la contradicción en la que se desarrolló el proyecto político dada la intromisión de la iglesia dentro de la base gubernamental. A pesar de lo anterior, el pensamiento científico dentro de la estructura social y educativa, que es una de las bases de la modernidad, fue instaurado en este periodo garciano.

Por tanto, y siguiendo el análisis de Echeverría para el caso de estudio, se puede aseverar, en primer lugar, que las bases de la modernidad en la ciudad de Quito se cimentaron desde el período garciano con la implementación de leyes y la construcción de infraestructura que permitieron que el pensamiento científico y académico se constituyeran como parte de la vida nacional. En segundo lugar, partiendo de la secularización de lo político que empieza con la revolución liberal a través de la declaración del estado laico, se refuerza el cambio a través de la construcción de un marco legal de respaldo, así como la construcción de más infraestructura que profundiza la transición hacia la modernidad; de hecho consideramos que la edificación icónica del cambio de pensamiento, en la ciudad de Quito, es la construcción del colegio Mejía como la primera institución educativa laica del país, que sin lugar a dudas da muestra clara de la separación de la iglesia del modelo de gobierno hasta entonces vigente. En tercer lugar, el inicio de la época liberal y sus efectos posteriores nos permiten apreciar como el pensamiento del individualismo tanto personal y colectivo se instaura claramente en los años posteriores a la posguerra y dio paso a grandes logros sociales como la expedición del Código del Trabajo (1937).

En el caso de la materialización o particularización misma de la modernidad en la ciudad de Quito, el modernismo como lo entiende Harvey empieza a evidenciarse durante la época de la revolución liberal (1895-1912) y se fortalece con el paso del tiempo, en ese contexto y sin lugar a dudas la llegada del ferrocarril a la ciudad constituye uno de los momentos destacados ya que ese hecho por si mismo significo o provocó uno de los cambios más importantes en la ciudad como es el cambio en los modos de producción. Es importante señalar que el cambio de modelo económico del país tuvo su motor en la costa, particularmente en la ciudad de Guayaquil y que el modelo de producción y consumo en la ciudad de Quito solo cambió al terminar con el aislamiento en el que vivía esta ciudad hasta antes de la llegada del ferrocarril. Este hecho es el que promovió un cambio a la

\footnotetext{
${ }^{14}$ El articulo está publicado en el libro Los hechos de la arquitectura (Pérez Oyarzún, Aravena, \& Quintanilla Chalá, 2002). Se refiere precisamente al pensamiento y el significado de la arquitectura moderna.
} 
urbe quiteña que comienza a crecer trascendiendo los límites del Centro Histórico.

En ese contexto si consideramos que para D. Harvey el modernismo se materializa a través de la máquina, la fábrica y la urbanización, podemos señalar que, de acuerdo con los sucesos históricos, las tres está estrechamente relacionadas toda vez que aparecieron casi simultáneamente en la ciudad de Quito. Paralelamente, se gestan cambios en los medios de comunicación en la ciudad con la construcción del ferrocarril, así como del edificio de los correos, la fundación del diario El Comercio (1906), entre otros; propiciando el crecimiento económico de la ciudad y la dinamización del comercio.

No hay que pasar por alto que el desarrollo económico de Quito, por su condición de ciudad capital, está relacionado directamente a grandes latifundistas que controlaban el territorio y en consecuencia el crecimiento de la ciudad se localiza en terrenos en los que se construyeron los barrios de expansión posterior a la llegada del ferrocarril entre los que se encuentran como el barrio América, la Magdalena y la Mariscal. Espacios que fueron comprados por el gobierno, beneficiando a sus propietarios no solo por la ganancia económica que les significo, sino además por las fuertes inversiones que realizo el mismo gobierno a través de la construcción de la infraestructura necesaria para servir a éstos y que fue entregada a la municipalidad sin un aporte significativo de los vendedores.

Desde una perspectiva arquitectónica, los cambios en la ciudad durante este periodo estuvieron marcados por un eclecticismo en donde las primeras manifestaciones modernas empiezan a evidenciarse. El impulso del comercio provocó los primeros cambios de uso y por tanto de morfología de expresión moderna, como es el caso de los pasajes comerciales en los que la apertura de la planta baja de algunas construcciones dio cabida a las vitrinas de exposición, como fue el caso del Pasaje Royal. Otro ejemplo destacado lo constituye el Antiguo Mercado de San Millán, actual Palacio de Cristal, que fue la primera estructura prefabricada de acero de la ciudad y que lo hace un elemento relevante dentro de la historia; a partir de entonces se empezó a construir con elementos de acero, primordialmente en cubiertas para estos mismos pasajes.

Por su parte, la introducción del hormigón armado, como expresión tecnológica estructural, no solo se dilato en su uso, sino que además sucedió en momentos diferentes entre Guayaquil y Quito.
En el caso de Guayaquil la primera construcción con esta técnica apareció ya en 1905, construcción de la Cárcel Municipal, mientras que en Quito fue hasta 1939 con la construcción del Hotel Humboldt. De esta manera se evidencia claramente como la influencia del ferrocarril es el detonante para el desarrollo tecnológico propio de la modernidad en la ciudad de Quito, ya que se expresa posterior a éste. No solo en la arquitectura que es punto de interés de este estudio sino en otras expresiones sociales, culturales y principalmente económicas.

Pero además el cambio morfológico también se hizo evidente en las viviendas una vez que los límites urbanos trascendieron el Centro Histórico, sobre todo, hacia La Mariscal donde empiezan a construir con retiros tanto frontales como laterales, aislando las construcciones, a diferencia de la configuración continua y en línea de fábrica propia del centro.

Finalmente, si bien las bases de pensamiento moderno fueron cimentadas en la etapa estudiada en este artículo, que van desde el mandato garciano, la revolución liberal y el posliberalismo, la expresión de la arquitectura moderna no se evidenció salvo algunas primeras manifestaciones como las que se mencionaron anteriormente. Por nuestra parte consideramos que el desarrollo teórico, tecnológico y morfológico de la modernidad en la arquitectura comienzan a establecerse a fines de la década de los treinta. De esta manera se puede concluir que la expresión misma del movimiento moderno no se evidenció en esta etapa como expresión misma de la arquitectura, pero sus bases fueron cimentadas, principalmente por los cambios de comportamiento como lo describe Echeverría y, sobre todo, porque los elementos materiales son claramente identificables tal como lo describe Harvey. Es por este motivo que el artículo pretende definir a este momento como de expresión protomoderna que es lo mismo que las bases de la modernidad. C

\section{REFERENCIAS BIBLIOGRÁFICAS}

Ayala Mora, E. (2008). Resumen de historia del
Ecuador. Quito: Corporación Editora
Nacional.
Aymonino, C. (1972). Orígenes y desarrollo de la
ciudad moderna. Barcelona: Gustavo Gili.
Bauman, Z. (1997). La posmodernidad y sus
descontentos. Madrid: Akal.
Benavides Solís, J. (1995). Arquitectura del siglo


$X X$ en Quito. Quito: Banco Central del Ecuador.

Benjamin, W. (1934). La obra de arte la época de su reproductibilidad técnica. México: Itaca.

Bienal de Arquitectura de Quito. (2018). Centro Cultural Itchimbía. Obtenido de Bienal Panamericana de Arquitectura de Quito BAQ 2018: http://baq-cae.ec/centrocultural-itchimbia/

del Pino, I. (1993). Sobre la arquitectura quiteña: 1820-1922. En I. Municipio de Quito, Arquitectura de Quito una vision histórica (págs. 115-136). Quito: Trama.

Echeverría, B. (2009). ¿Qué es la modernidad? México: UNAM.

Empresa Pública Municipal de Turismo, Promoción Cívica y Relaciones Internacionales de Guayaquil, EP. (2018). Antigua Cárcel Municipal (Patrimonial). Obtenido de Guayaquil es mi destino: http://www. guayaquilesmidestino.com/es/patrimoniode-la-ciudad/edificios-publicos/antiguacarcel-municipal

Empresa Pública Municipal de Turismo, Promoción Cívica y Relaciones Internacionales de Guayaquil, EP. (2018). Antiguo Mercado Sur "Palacio de Cristal" (Patrimonial). Obtenido de Guayaquil es mi destino: http://www.guayaquilesmidestino.com/es/ patrimonio-de-la-ciudad/edificios-publicos/ palacio-de-cristal

Fernández Cox, C. (1991). Modernidad apropiada. En C. Fernández Cox, E. Browne, C. E. Comas, R. Santa María, F. Liernur, A. Dewes, \& M. Waisman, Modernidad y posmodernidad en América Latina (págs. 11-22). Bogotá: Escala.

González, M., Sosa, C., \& Merino, G. (1993). Conformación y transformaciones urbano arquitectónicas del sector Mariscal Sucre (1922-1988). En I. M. Quito, Arquitectura de Quito. Una visión Histórica (págs. 153172). Quito: Trama.

Habermas, J. (1997). Arquitectura moderna y posmoderna. En N. Leach, Rethinkg Architecture (págs. 225-235). New York: Routledge.

Harvey, D. (1990). La condición de la posmodernidad. Buenos Aires: Amorrortu.

I. Municipio del Distrito Metropolitano de Quito. (2004). Guia de Arquitectura de Quito. Quito: Junta de Andalucía.
I. Municipio del Distrito Metropolitano de Quito. (2017). Archivo Histórico. Obtenido de Secretaría de Territorio. Alcaldía de Quito: http://sthv.quito.gob.ec/archivo-historico/

Ilustre Municipio del Distrito Metropolitano de Quito. (s.f.). Instituto Metropolitano de Patrimonio. Obtenido de Inventario Patrimonial: http://www.patrimonio.quito. gob.ec/index.php/patrimonio-cultural/ inventario-patrimonial

Instituto Nacional de Patrimonio Cultural. (2015). Pasaje Royal Quito. Obtenido de Fotografía Patrimonial: http://www. fotografianacional.gob.ec/web/en/galeria/ element/7971

Loor, J. M. (17 de septiembre de 2017). La arquitectura moderna en el inventario patrimonial de Quito. (S. Camacho, Entrevistador)

Maldonado, C. (1993). La arquitectura de Quito en la época republicana. En I. M. Quito, Arquitectura de Quito. Una visión histórica (págs. 137-152). Quito: Trama.

Maluenda, A. E. (2016). La arquitectura moderna en Latinoamérica. Barcelona: Reverté.

Mann, M. (1991). Las fuentes del poder social. Tomo I. Madrid: Alianza.

Masiero, R. (2003). Estética de la arquitectura. Madrid: Machado Libros S.A.

Moreira, R., \& Álvarez , Y. (2004). Arqutiectura de Quito 1915-1985. Quito: TRAMA.

Pérez Oyarzún, F., Aravena, A., \& Quintanilla Chalá, J. (2002). Los hechos de la Arquitectura. Santiago: Ediciones ARQ/ Pontificia Universidad Católica de Chile.

Segre, R. (1975). América Latina en su arquitectura. México: Siglo XXI.

Treviño Moreno, P. (2000). Apuntes para una definición de la modernidad. En Z Zeraoui, Modernidad y posmodernidad (págs. 9-25). México: Noriega.

Zeraoui, Z. (2000). Modernidady posmodernidad. México: Noriega. 